\title{
LOOP SPACES OF FINITE COMPLEXES AT LARGE PRIMES
}

\author{
C. A. MCGIBBON AND C. W. WILKERSON ${ }^{1}$
}

\begin{abstract}
Let $X$ be a finite, simply connected CW complex with only a finite number of nonzero rational homotopy groups. Localized away from a certain finite set of primes, the loop space of $X$ is shown to be homotopy equivalent to a product of spheres and loop spaces of spheres. Applications to the homotopy groups of $X$ and the homological properties of $\Omega X$ are given.
\end{abstract}

Let $X$ be a simply connected CW complex with a finite numer of cells. Define the exponent of $X$ at a prime $p$ to be the least upper bound of the orders of $p$-primary torsion elements in the homotopy groups, $\pi_{*} X$.

Is this exponent finite or not? The following conjecture, due to J. C. Moore [2], asserts that the answer depends only on a certain rational homotopy invariant of $X$.

Conjecture. Given $X$ as above, the exponent of $X$ at $p$ is finite if and only if the total rational homotopy $\operatorname{rank}, \sum_{n} \operatorname{rank}\left(\pi_{n} X \otimes \mathbf{Q}\right)$, is finite.

This conjecture was the starting point for the results in this paper. We prove one direction of this conjecture for all but a finite number of primes. Our main geometric result is the following.

THEOREM. Let $X$ be a finite, 1-connected, $C W$ complex whose total rational homotopy rank is finite and nonzero. Then for almost all primes $p$, the loop space $\Omega X$ is p-equivalent to a product of spheres and loop spaces of spheres; that is

$$
\Omega X \simeq_{p} \prod_{i} S^{2 m_{i}-1} \times \prod_{j} \Omega S^{2 n_{j}-1} .
$$

Special cases of this result have been known for some time. A case in point is the splitting

$$
\Omega S^{2 n} \simeq_{p} S^{2 n-1} \times \Omega S^{4 n-1},
$$

valid for each odd prime $p$. The number of factors in such splittings is easily seen to equal the total rational homotopy rank of $X$.

The sphere $S^{n}$ is known to have a finite exponent at each prime. For $p=2$ this is due to I. M. James [4]; for odd primes it is due to H. Toda [5]. Consequently Moore's exponent conjecture is true for all spaces and primes covered by our theorem. Here are some other immediate algebraic consequences.

Received by the editors April 8, 1985.

1980 Mathematics Subject Classification. Primary 55P35; Secondary 55Q52.

Key words and phrases. Loop spaces, homotopy group, exponents in homotopy groups, localization of homotopy types.

${ }^{1}$ Research partially supported by the NSF and Wayne State Fund. 
COROLLARY. Let $X$ be as in the theorem. Then for almost all primes $p$,

(i) $H_{*}(\Omega X ; \mathbf{Z})$ has no $p$-torsion,

(ii) pth powers vanish in $\tilde{H}^{*}(\Omega X ; \mathbf{Z} / p)$,

(iii) the Steenrod algebra acts trivially on $H^{*}(\Omega X ; \mathbf{Z} / p)$.

It would be interesting to know the extent to which this corollary holds for loop spaces of finite complexes of infinite rational homotopy rank. Property (ii), which is equivalent to primitive generation of the homology Hopf algebra, does appear to hold in general. Moreover if $X$ is the suspension of a finite complex, then the loop space of $X$ has these properties for almost all primes.

On the other hand, we now know that the first property does not hold in general. Recently, D. Anick [1] has constructed a finite complex $K$ which dramatically illustrates the failure of (i) for general $X$. The example $K$ has the form $\bigvee^{7} S^{2} \cup \bigvee^{11} e^{4}$ and yet $H_{*}(\Omega K, \mathbf{Z})$ has torsion of every possible order! Indeed for each positive integer $m$ it has torsion of order $m$ in degree $m+1$.

We thank Bill Dwyer and Steve Halperin for their help and guidance in the subjects considered in this paper.

PROOF OF THE MAIN THEOREM. We shall consider not only finite complexes but also those localizations of finite complexes in which only a finite number of primes have been inverted. Our result adapts easily to this slightly more general situation.

The proof uses induction on the total rational homotopy rank. The first case (i.e., rank $=1$ ) is easily settled because the only possibility here is that $X \simeq_{p} S^{2 n-1}$ for almost all $p$.

In the induction step, assume that $X$ is a 1-connected, finite localization of a finite complex with rational rank $n \geq 2$. Take $k$ to be the smallest integer such that $\pi_{k} X \otimes \mathbf{Q} \neq \mathbf{0}$. The Hurewicz homomorphism is a rational isomorphism in this dimension and so there is a nonzero homomorphism

$$
f: H_{k}(X ; \mathbf{Z}) \rightarrow \mathbf{Q} .
$$

Let $L \subset \mathbf{Q}$ denote the image of $f$. The finiteness conditions on $X$ imply that $L$ is a finite localization of the integers.

These homomorphisms determine cohomology classes in the usual manner and hence give rise to the following diagram of maps into Eilenberg-Mac Lane spaces:

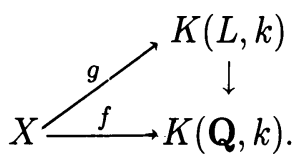

Here the bottom map corresponds to the homomorphism of the same name mentioned earlier. The inclusion $L \subset \mathbf{Q}$ induces the corresponding vertical map.

Now assume that $k$ is odd and consider the lifting problem:

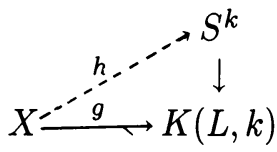

The vertical map $j$ is chosen so as to induce an isomorphism on $H_{k}(-; L)$. For almost all primes $p$, this map will induce an equivalence at $p$ through a range of 
dimensions that exceed that of $X$. Hence a lift $h$ exists after at most a finite localization. It is easy to check that this lift must be rationally essential. Indeed, it induces a rational epimorphism onto $\pi_{k} S^{k}$.

We now convert this map $h$ into a fibration and study its fiber:

$$
F \rightarrow X \stackrel{h}{\rightarrow} S^{k}
$$

In order to apply the induction hypothesis to $F$, we need to show that this fiber satisfies our finiteness requirements. By Halperin $[3], F$ has the rational homotopy type of a finite complex. Therefore, the question of finiteness can be resolved by examining the torsion in $H_{*}(F ; \mathbf{Z})$. To do this, we use the principal fibration $\Omega S^{k} \rightarrow F \rightarrow X$, induced by $h$. The corresponding action $\Omega S^{k} \times F \rightarrow F$ gives $H_{*}(F ; \mathbf{Z})$ the structure of a module over the Pontryagin ring $H_{*}\left(\Omega S^{k} ; \mathbf{Z}\right)$. Call this ring $R$ for the moment. We claim that as an $R$-module, $H_{*}(F ; \mathbf{Z})$ is finitely generated.

To see this note that the spaces in this principal fibration are simply connected. We can assume that they each have been localized away from the same finite set of primes. This set can be taken large enough so that $H_{*}(X ; \mathbf{Z})$ may be assumed to be torsion free.

In the Serre spectral sequence for this fibration, we then have

$$
E_{p, *}^{2} \approx H_{p}(X ; \mathbf{Z}) \otimes \mathbf{R} .
$$

Thus it follows from the finiteness of $X$ that this $E^{2}$-term is a finitely generated $R$-module. Now this ring $R$, being polynomial on a single generator, is noetherian. Over such rings finite generation is inherited by submodules and quotients. Thus every term, $E^{r}$, in the spectral sequence is likewise finitely generated. Since $X$ is finite dimensional we have, of course, $E^{r}=E^{\infty}$ for $r$ sufficiently large. Now $E^{\infty}$ is the graded object of a complete filtration of $H_{*}(F ; \mathbf{Z})$ by $R$-modules. Consequently the finite generation of $H_{*}(F ; \mathbf{Z})$ follows from that of $E^{\infty}$.

The elements of finite order in $H_{*}(F ; \mathbf{Z})$ form an $R$-submodule, and as such, it must be finitely generated. This implies that $p$-torsion can occur in $H_{*}(F ; \mathbf{Z})$ for at most a finite number of primes.

Localize $F$ away from those torsion primes just considered. The result (in view of Halperin's determination of the rational type) is the finite localization of a finite complex.

Return now to the original fibration

$$
F \rightarrow X \stackrel{h}{\rightarrow} S^{k}
$$

We have shown that after a finite localization the fiber $F$ satisfies our finiteness requirements. We may also assume that there is a section (again, after a finite localization if necessary). Having made these localizations we obtain the splitting of loop spaces,

$$
\Omega X \simeq \Omega F \times \Omega S^{k} .
$$

The rational rank of $F$ is one less than that of $X$ and so $\Omega F$ splits as required by the induction hypothesis. This completes the induction step when $k$ is odd. 
The even case (say $k=2 n$ ) is easier; there we begin with the lifting problem

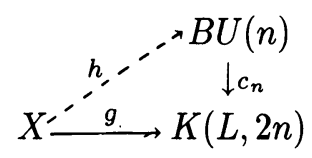

wherein the bottom line occurs just as before and the vertical map is induced by the $n$th Chern class.

Let $d$ denote the dimension of $X$. Localized at most primes (for example $p>d$ ), the $d$-skeleton of $B U(n)$ contains the $d$-skeleton of $K(L, 2 n)$ as a retract. Moreover, the restriction of $c_{n}$ can be taken to be the retraction in question. Hence this lifting problem has a solution $h$, after at most a finite localization. In addition, this lift $h$ induces a rational epimorphism on $\pi_{2 n}()$.

Now consider the pullback diagram of fibrations:

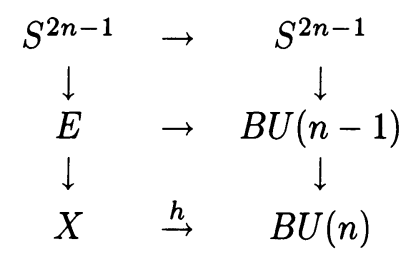

Apply $\pi_{*}() \otimes \mathbf{Q}$ to this diagram and observe that the rational rank of $E$ is one less than that of $X$. The finiteness of $E$ is, of course, immediate.

A glance at the induced fibration $\Omega E \rightarrow \Omega X \rightarrow S^{2 n-1}$ shows that it has a section after at most a finite localization. With this accomplished we have

$$
\Omega X \simeq \Omega E \times S^{2 n-1} .
$$

Once again the induction hypothesis implies that $\Omega E$ splits are required and the proof is complete.

Given a space $X$ that satisfies the hypothesis of our theorem, let $P(X)$ denote the finite set of exceptional primes; that is, those primes $p$ at which $\Omega X$ does not decompose in the manner described by our theorem. It would be interesting to know more about this set. To seek a precise description of $P(X)$ seems unrealistic; indeed for $X=S^{2 n}$ this is equivalent to the Hopf invariant one problem. It would be more reasonable to look for upper bounds on $P(X)$. Unfortunately our proof does not shed much light on this problem. In particular, in the induction step for $k$ odd, the Noetherian argument gives no description of $P(X)$, beyond its finiteness. So consider instead the following three examples:

$X_{1}=$ the product $S^{n} \times$ (Moore space),

$X_{2}=$ the Lie group $S U(n)$,

$X_{3}=$ the $2 m n$-skeleton of $\Omega S^{2 n+1}$.

All three spaces satisfy the hypothesis of our theorem. The first shows that $P(X)$ can contain the torsion primes in $H_{*}(X ; \mathbf{Z})$. The second shows that $P(X)$ can contain the torsion primes in the cokernel of the Hurewicz homomorphism. In the third example, $P\left(X_{3}\right) \supseteq[p \mid p \leq m]$ and hence depends in part upon the dimension of $X$. Is it possible to obtain an upper bound for $P(X)$ in terms of the three ingredients just mentioned? If not, what else is needed? 


\section{REFERENCES}

1. D. J. Anick, A loop space whose homology has torsion of all orders, preprint, 1985.

2. F. R. Cohen, J. C. Moore and J. A. Neisendorfer, Torsion in homotopy groups, Ann. of Math. (2) 109 (1979), 121-168.

3. S. Halperin, Finiteness in the minimal models of Sullican, Trans. Amer. Math. Soc. 230 (1977), 173-199.

4. I. M. James, On the suspension sequence, Ann. of Math. (2) 65 (1957), 74-107.

5. H. Toda, On the double suspension $E^{2}$, J. Inst. Polytech. Osaka City Univ. Ser. A 7 (1956), 103-145.

Department of Mathematics, Wayne State University, Detroit, Michigan 48202 